\title{
Discrete Pseudo-Differential Operators and Boundary Value Problems in a Half-Space and a Cone
}

\author{
V. Vasilyev* \\ (Submitted by E. K. Lipachev) \\ Chair of Differential Equations, Belgorod National Research University, \\ Studencheskaya 14/1, Belgorod, 308007 Russia \\ Received June 26, 2016
}

\begin{abstract}
We consider a certain class of discrete pseudo-differential operators and related equations in a sharp convex cone and describe their invertibility conditions in $L_{2}$ spaces. For this purpose we introduce a concept of periodic wave factorization for elliptic symbol and show its applicability for the studying. For a half-space case we consider the Laplace equation and describe a solution of the discrete Dirichlet problem.
\end{abstract}

DOI: $10.1134 / \mathrm{S} 1995080218020270$

Keywords and phrases: Discrete operator, multidimensional periodic Riemann problem, periodic wave factorization, invertibility, discrete boundary condition.

\section{INTRODUCTION}

A classical pseudo-differential operator in Euclidean space $\mathbb{R}^{m}$ is defined by the formula [1-4]

$$
(A u)(x)=\iint_{\mathbb{R}^{m}} \int_{\mathbb{R}^{m}} \widetilde{A}(x, \xi) e^{i(\xi-y)} \tilde{u}(\xi) d \xi d y,
$$

where the sign $\sim$ over a function denotes its discrete Fourier transform

$$
\widetilde{u}(\xi)=\int_{\mathbb{R}^{m}} u(x) e^{i x \cdot \xi} d x .
$$

Given function $u_{d}$ of a discrete variable $\tilde{x} \in \mathbb{Z}^{m}$ we define its discrete Fourier transform by the series

$$
\left(F_{d} u_{d}\right)(\xi) \equiv \widetilde{u}_{d}(\xi)=\sum_{\tilde{x} \in \mathbb{Z}^{m}} e^{i \tilde{x} \cdot \xi} u(\tilde{x}), \quad \xi \in \mathbb{T}^{m} \equiv[-\pi, \pi]^{m}
$$

where partial sums are taken over cubes $Q_{N}=\left\{\tilde{x} \in \mathbb{Z}^{m}: \tilde{x}=\left(\tilde{x}_{1}, \cdots, \tilde{x}_{m}\right), \max _{1 \leq k \leq m}\left|\tilde{x}_{k}\right| \leq N\right\}$.

Let $D \subset \mathbb{R}^{m}$ be a sharp convex cone, $D_{d} \equiv D \cap \mathbb{Z}^{m}$, and $L_{2}\left(D_{d}\right)$ be a space of functions of discrete variable defined on $D_{d}$, and $A(\tilde{x})$ be a given function of a discrete variable $\tilde{x} \in \mathbb{Z}^{m}$. We introduce the function $\widetilde{A}_{d}(\xi)=\sum_{\tilde{x} \in \mathbb{Z}^{m}} e^{i \tilde{x} \cdot \xi} A(\tilde{x}), \xi \in \mathbb{T}^{m}$, and consider the following types of operators

$$
\left(A_{d} u_{d}\right)(\tilde{x})=\int_{\mathbb{T}^{m}} \sum_{\tilde{y} \in D_{d}} e^{i(\tilde{y}-\tilde{x}) \cdot \xi} \widetilde{A}(\xi) \tilde{u}_{d}(\xi) d \xi, \quad \tilde{x} \in D_{d}
$$

according to a standard definition.

Definition 1. The function $\widetilde{A}_{d}(\xi)$ is called a symbol of the operator $A_{d}$, and this symbol is called an elliptic symbol if $\tilde{A}_{d}(\xi) \neq 0, \forall \xi \in \mathbb{T}^{m}$.

\footnotetext{
${ }^{*}$ E-mail: vbv57@inbox.ru
} 
Our main goal is describing a periodic variant of wave factorization for an elliptic symbol [10] and showing its usability for studying invertibility for the operator $A_{d}$.

Let us denote $P_{D_{d}}$ projection operator on $D_{d}, P_{D_{d}}: L_{2}\left(\mathbb{Z}^{m}\right) \rightarrow L_{2}\left(D_{d}\right)$ so that for arbitrary function $u_{d} \in L_{2}\left(\mathbb{Z}^{m}\right)$

$$
\left(P_{D_{d}} u_{d}\right)(\tilde{x})= \begin{cases}u_{d}(\tilde{x}), & \text { if } \tilde{x} \in D_{d} \\ 0, & \text { otherwise }\end{cases}
$$

If we consider a half-space case then the Fourier image of the operator $P_{D_{d}}$ is evaluated $[12,13]$ and we'll demonstrate it in the following

Example 1. If $D=\mathbb{R}_{+}^{m}$ then

$$
\left(F_{d} P_{D_{d}} u_{d}\right)\left(\xi^{\prime}, \xi_{m}\right)=\frac{1}{4 \pi i} \lim _{\tau \rightarrow 0+} \int_{-\pi}^{\pi} u_{d}\left(\xi^{\prime}, \eta_{m}\right) \cot \frac{\xi_{m}-\eta_{m}+i \tau}{2} d \eta_{m} .
$$

If $D$ is a sharp convex cone $C_{+}^{a}=\left\{\tilde{x} \in \mathbb{Z}^{m}: \tilde{x}=\left(\tilde{x}_{1}, \cdots, \tilde{x}_{m}\right), \tilde{x}_{m}>a\left|\tilde{x}^{\prime}\right|, \tilde{x}^{\prime}=\left(\tilde{x}_{1}, \cdots, \tilde{x}_{m-1}\right)\right.$, $a>0\}$ then we introduce the function $B_{d}(z)=\sum_{\tilde{x} \in D_{d}} e^{i \tilde{x} \cdot z}, z=\xi+i \tau, \xi \in \mathbb{T}^{m}, \tau \in C_{+}^{a}$, and define the operator

$$
\left(B_{d} u\right)(\xi)=\lim _{\tau \rightarrow 0} \int_{\mathbb{T}^{m}} B_{d}(z-\eta) u_{d}(\eta) d \eta
$$

Lemma 1. For arbitrary $u_{d} \in L_{2}\left(\mathbb{Z}^{m}\right)$ the property $F_{d} P_{D_{d}} u_{d}=B_{d} F_{d} u_{d}$ holds.

Proof. Let $\chi_{+}(\tilde{x})$ be an indicator of the set $D_{d}$. Thus $\left(P_{D_{d}} u_{d}\right)(\tilde{x})=\chi_{+}(\tilde{x}) \cdot u_{d}(\tilde{x})$. Further since the function $\chi_{+}(\tilde{x})$ is not summarizable we can't apply directly a convolution property of the Fourier transform. We choose the function $e^{i \tilde{x} \cdot \tau}$ so the product $\chi_{+}(\tilde{x}) e^{i \tilde{x} \cdot \tau}$ will be summarizable for some admissible $\tau$. Taking into account a forthcoming passing to a limit under $\tau \rightarrow 0+$ we have $F_{d}\left(\chi_{+}(\tilde{x}) e^{i \tilde{x} \cdot \tau}\right)=B_{d}(z)$. Thus we can use the Fourier transform obtaining convolution of functions $B_{d}(z)$ and $\widetilde{u}_{d}(\xi)$. It is left passing to a limit.

\section{MULTIDIMENSIONAL PERIODIC RIEMANN BOUNDARY VALUE PROBLEM}

For $D=\mathbb{R}_{+}^{m}$ we'll remind some author's constructions [12,13] for discrete equations in a half-space. We have $B_{d}(z)=\cot \frac{z}{2}, z=\left(\xi^{\prime}, \xi_{m}+i \tau\right), \xi^{\prime}=\left(\xi_{1}, \cdots, \xi_{m-1}\right), \tau>0$. Thus (see Example 1) we use a periodic one-dimensional Riemann problem with a parameter $\xi^{\prime} \in \mathbb{T}^{m-1}$ which is the following. Finding a pair of functions $\Phi^{ \pm}\left(\xi^{\prime}, \xi_{m}\right)$ which are boundary values of holomorphic in half-strips $\Pi_{ \pm}=\{z \in \mathbb{C}$ : $\left.z=\xi_{m} \pm i \tau, \tau>0\right\}$ such that these are satisfied a linear relation

$$
\Phi^{+}(\xi)\left(\xi^{\prime}, \xi_{m}\right)=G\left(\xi^{\prime}, \xi_{m}\right) \Phi^{-}(\xi)\left(\xi^{\prime}, \xi_{m}\right)+g(\xi), \quad \xi \in \mathbb{T}^{m},
$$

for almost all $\xi^{\prime} \in \mathbb{T}^{m-1}$, where $G(\xi), g(\xi)$ are given periodic functions.

Now we will consider an essential multidimensional case. Let $\stackrel{*}{D}$ be a conjugate cone for $D$ i.e. $\stackrel{*}{D}=\left\{x \in \mathbb{R}^{m}: x \cdot y>0, y \in D\right\}$, and $T(\stackrel{*}{D}) \subset \mathbb{C}^{m}$ be a set of the type $\mathbb{T}^{m}+i \stackrel{*}{D}$. For $\mathbb{T}^{m} \equiv \mathbb{R}^{m}$ such a domain of multidimensional complex space is called a radial tube domain over the cone $D^{*}[7,8,10]$.

Let us define the subspace $A\left(\mathbb{T}^{m}\right) \subset L_{2}\left(\mathbb{T}^{m}\right)$ consisting of functions which admit a holomorphic continuation into $T(\stackrel{*}{D})$ and satisfy the following condition

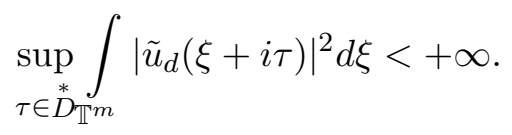

In other words the space $A\left(\mathbb{T}^{m}\right) \subset L_{2}\left(\mathbb{T}^{m}\right)$ consists of boundary values of holomorphic in $T(\stackrel{*}{D})$ functions. 
Let us denote $B\left(\mathbb{T}^{m}\right)=L_{2}\left(\mathbb{T}^{m}\right) \ominus A\left(\mathbb{T}^{m}\right)$, so that $B\left(\mathbb{T}^{m}\right)$ is a direct complement of $A\left(\mathbb{T}^{m}\right)$ in $L_{2}\left(\mathbb{T}^{m}\right)$. A simplest variant of this problem is so called a jump problem. We formulate the problem by the following way: finding a pair of functions $\Phi^{ \pm}, \Phi^{+} \in A\left(\mathbb{T}^{m}\right), \Phi^{-} \in B\left(\mathbb{T}^{m}\right)$, such that

$$
\Phi^{+}(\xi)-\Phi^{-}(\xi)=g(\xi), \quad \xi \in \mathbb{T}^{m},
$$

where $g(\xi) \in L_{2}\left(\mathbb{T}^{m}\right)$ is given.

Lemma 2. The operator $B_{d}: L_{2}\left(\mathbb{T}^{m}\right) \rightarrow A\left(\mathbb{T}^{m}\right)$ is a bounded projector. A function $u_{d} \in L_{2}\left(D_{d}\right)$ iff its Fourier transform $\tilde{u}_{d} \in A\left(\mathbb{T}^{m}\right)$.

Proof. According to standard properties of the discrete Fourier transform $F_{d}$ we have

$$
F_{d}\left(\chi_{+}(\tilde{x}) u_{d}(\tilde{x})\right)=\lim _{\tau \rightarrow 0} \int_{\mathbb{T}^{m}} B_{d}(z-\eta) \widetilde{u}_{d}(\eta) d \eta,
$$

where $\chi_{+}(\tilde{x})$ is an indicator of the set $D_{d}$. It implies a boundedness of the operator $B_{d}$. The second assertion follows from holomorphic properties of the kernel $B_{d}(z)$. In other words for arbitrary function $v \in A\left(\mathbb{T}^{m}\right)$ we have

$$
v(z)=\int_{\mathbb{T}^{m}} B_{d}(z-\eta) v(\eta) d \eta, \quad z \in T(\stackrel{*}{D}) .
$$

It is an analogue of the Cauchy integral formula.

Theorem 1. The jump problem has unique solution for arbitrary right-hand side from $L_{2}\left(\mathbb{T}^{m}\right)$.

Proof. Indeed it is equivalent to one-to-one representation of the space $L_{2}\left(D_{d}\right)$ as a direct sum of two subspaces. If we'll denote $\chi_{+}(x), \chi_{-}(x)$ indicators of discrete sets $D_{d}, \mathbb{Z}^{m} \backslash D_{d}$ respectively then the following representation $u_{d}(\tilde{x})=\chi_{+}(\tilde{x}) u_{d}(\tilde{x})+\chi_{-}(\tilde{x}) u_{d}(\tilde{x})$ is unique and holds for arbitrary function $u_{d} \in L_{2}\left(\mathbb{Z}^{m}\right)$. After applying the discrete Fourier transform we have $F_{d} u_{d}=F_{d}\left(\chi_{+} u_{d}\right)+F_{d}\left(\chi_{-} u_{d}\right)$, where $F_{d}\left(\chi_{+} u_{d}\right) \in A\left(\mathbb{T}^{m}\right)$ according to Lemma 2 , and thus $F_{d}\left(\chi_{-} u_{d}\right)=F_{d} u_{d}-F_{d}\left(\chi_{+} u_{d}\right) \in B\left(\mathbb{T}^{m}\right)$ because $F_{d} u_{d} \in L_{2}\left(\mathbb{T}^{m}\right)$.

Example 2. If $m=2$ and $C_{+}^{2}$ is the first quadrant in a plane then a solution of a jump problem is given by formulas

$$
\begin{gathered}
\Phi^{+}(\xi)=\frac{1}{(4 \pi i)^{2}} \lim _{\tau \rightarrow 0} \int_{-\pi}^{\pi} \int_{-\pi}^{\pi} \cot \frac{\xi_{1}+i \tau_{1}-t_{1}}{2} \cot \frac{\xi_{2}+i \tau_{2}-t_{2}}{2} g\left(t_{1}, t_{2}\right) d t_{1} d t_{2}, \\
\Phi^{-}(\xi)=\Phi^{+}(\xi)-g(\xi), \quad \tau=\left(\tau_{1}, \tau_{2}\right) \in C_{+}^{2} .
\end{gathered}
$$

Now we can formulate a general statement for the multidimensional periodic Riemann boundary value problem. It looks as follows. Finding a pair of functions $\Phi^{ \pm}, \Phi^{+} \in A\left(\mathbb{T}^{m}\right), \Phi^{-} \in B\left(\mathbb{T}^{m}\right)$, such that

$$
\Phi^{+}(\xi)=G(\xi) \Phi^{-}(\xi)+g(\xi), \quad \xi \in \mathbb{T}^{m},
$$

where $G(\xi), g(\xi)$ are given periodic functions. If $G(\xi) \equiv 1$ we have the jump problem (3).

Like classical studies $[5,6]$ we want to use a special representation for an elliptic symbol to solve the problem (4). We can easily obtain so-called characteristic singular integral equation associated with multidimensional periodic Riemann boundary value problem (4) like [5, 6] (see also [12, 13]).

Let us denote $Q_{D_{d}}=I-P_{D_{d}}$ and consider so-called paired operator composed by two operators $A_{d}^{(1)}, A_{d}^{(2)}$ of the following type

$$
A_{d}^{(1)} P_{D_{d}}+A_{d}^{(2)} Q_{D_{d}}: L_{2}\left(\mathbb{Z}^{m}\right) \rightarrow L_{2}\left(\mathbb{Z}^{m}\right) .
$$

One can easily obtain the following

Lemma 3. The invertibility of the operator (1) in the space $L_{2}\left(D_{d}\right)$ is equivalent to invertibility of the operator (5) in the space $L_{2}\left(\mathbb{Z}^{m}\right)$ with $A_{d}^{(1)}=A_{d}, A_{d}^{(2)}=I$. 
The Fourier image for the operator (5) is the following operator

$$
\tilde{u}_{d}(\xi) \longmapsto\left(\left(A_{d}^{(1)}(\xi) B_{d}+A_{d}^{(2)}(\xi)\left(I-B_{d}\right) \tilde{u}_{d}\right)(\xi) .\right.
$$

If $D=\mathbb{R}_{+}^{m}$ then the operator (6) is a one-dimensional singular integral operator with periodic Cauchy kernel and a parameter $\xi^{\prime}[12,13]$

\section{PERIODIC WAVE FACTORIZATION}

Definition 2. Periodic wave factorization for elliptic symbol $\tilde{A}(\xi)$ is called its representation in the form $\tilde{A}_{d}(\xi)=\tilde{A}_{\neq}(\xi) \tilde{A}_{=}(\xi)$, where the factors $A_{\neq}^{ \pm 1}(\xi), A_{\equiv}^{ \pm 1}(\xi)$ admit bounded holomorphic continuation into domains $T( \pm \stackrel{*}{D})$.

\subsection{Sufficient Conditions}

We'll give here certain sufficient conditions for an existence of the periodic wave factorization for an elliptic symbol.

Theorem 2. Let an elliptic symbol $\tilde{A}_{d}(\xi) \in C\left(\mathbb{T}^{m}\right)$ be a such that

$$
\begin{gathered}
\operatorname{supp} F_{d}^{-1}\left(\ln \tilde{A}_{d}(\xi)\right) \subset D_{d} \cup\left(-D_{d}\right), \\
\int_{-\pi}^{\pi} d \arg \tilde{A}_{d}\left(\cdots, \xi_{k}, \cdots\right)=0, \quad k=1, \cdots, m .
\end{gathered}
$$

Then the symbol $\tilde{A}_{d}(\xi)$ admits the wave factorization.

Proof. If we start from equality $\tilde{A}_{d}(\xi)=\tilde{A}_{\neq}(\xi) \tilde{A}_{=}(\xi)$ then by logarithm we obtain $\ln \tilde{A}_{d}(\xi)=$ $\ln \tilde{A}_{\neq}(\xi)+\ln \tilde{A}_{=}(\xi)$ and we have a special kind of a jump problem.

Namely if we'll denote by $A_{1}\left(\mathbb{T}^{m}\right)$ a subspace of the space $L_{2}\left(\mathbb{T}^{m}\right)$ consisting of functions which admit a holomorphic continuation into $T(-\stackrel{*}{D})$ and satisfy the condition $(2)$ for $\tau \in-\stackrel{*}{D}$. So evidently we speak on a possibility of decomposition of the function $\ln \tilde{A}_{d}(\xi)$ into two summands one of which belongs to the space $A\left(\mathbb{T}^{m}\right)$ and the second one belongs to the space $A_{1}\left(\mathbb{T}^{m}\right)$. Let us denote $F^{-1}\left(\ln \tilde{A}_{d}(\xi)\right) \equiv$ $v(x)$. If supp $v \subset D_{d} \cup\left(-D_{d}\right)$ then we have the unique representation $v=\chi_{+} v+\chi_{-} v$, where $\chi_{ \pm}$is an indicator of the discrete set $\pm D_{d}$. Further passing to the Fourier transform and potentiating we obtain the required factorization.

Remark 1. The condition (7) is not necessary but we have no an algorithm for constructing a periodic wave factorization. For $D=\mathbb{R}_{+}^{m}$ a such algorithm exists always (see [13]).

\subsection{Factorization and Index}

There is one point in previous considerations from proof of the Theorem 2 for which one needs an explanation. Indeed the function $\ln \tilde{A}(\xi)$ is defined correctly because the condition (8) provides an absence of bifurcation points. That's why one can call this factorization with vanishing index. 


\section{INVERTIBILITY OF DISCRETE OPERATORS}

Lemma 4. If $f \in B\left(\mathbb{T}^{m}\right), g \in A_{1}\left(\mathbb{T}^{m}\right)$ then $f \cdot g \in B\left(\mathbb{T}^{m}\right)$.

Proof. According to properties of discrete Fourier transform $F_{d}$ we have

$$
\left(F_{d}^{-1}(f \cdot g)\right)(\tilde{x})-\left(\left(F_{d}^{-1} f\right) *\left(F_{d}^{-1} g\right)\right)(\tilde{x}) \equiv \sum_{\tilde{y} \in \mathbb{Z}^{m}} f_{1}(\tilde{x}-\tilde{y}) g_{1}(\tilde{y})=\sum_{\tilde{y} \in-D_{d}} f_{1}(\tilde{x}-\tilde{y}) g_{1}(\tilde{y}),
$$

where $f_{1}=F_{d}^{-1} f, g_{1}=F_{d}^{-1} g$ and according to Lemma 2 supp $g_{1} \subset-D_{d}$. Further since we have supp $f_{1} \subset \mathbb{Z}^{m} \backslash\left(-D_{d}\right)$ then for $\tilde{x} \in D_{d}, \tilde{y} \in-D_{d}$ we have $\tilde{x}-\tilde{y} \in D_{d}$ so that $f_{1}(\tilde{x}-\tilde{y})=0$ for such $\tilde{x}$, $\tilde{y}$. Thus supp $f_{1} * g_{1} \subset \mathbb{Z}^{m} \backslash D_{d}$.

Theorem 3. If the elliptic symbol $\tilde{A}_{d}(\xi) \in C\left(\mathbb{T}^{m}\right)$ admits periodic wave factorization then the operator $A_{d}$ is invertible in the space $L_{2}\left(D_{d}\right)$.

Proof. We'll remind that according to the Lemma 3 an invertibility of the operator $A_{d}$ in the space $L_{2}\left(D_{d}\right)$ is equivalent to an invertibility of the operator $A_{d} P_{D_{d}}+I Q_{D_{d}}$ in the space $L_{2}\left(\mathbb{Z}^{m}\right)$. It is easily concluding the last invertibility is equivalent to solving the Riemann problem (4) for arbitrary right-hand side $g(\xi) \in L_{2}\left(\mathbb{Z}^{m}\right)$ with $G(\xi) \equiv \tilde{A}_{d}^{-1}(\xi)$. If we have the periodic wave factorization for the symbol $\tilde{A}_{d}(\xi)$ then

$$
\tilde{A}_{\neq}(\xi) \Phi^{+}(\xi)=\tilde{A}_{=}^{-1}(\xi) \Phi^{-}(\xi)+\tilde{A}_{\neq}(\xi) g(\xi), \quad \xi \in \mathbb{T}^{m},
$$

and we have a jump problem. The first summand $\tilde{A}_{\neq}(\xi) \Phi^{+}(\xi) \in A\left(\mathbb{T}^{m}\right)$ according to a holomorphic property, and the second one $\tilde{A}_{=}^{-1}(\xi) \Phi^{-}(\xi) \in B\left(\mathbb{T}^{m}\right)$ according to the lemma 3 . Taking into account the theorem 2 we conclude that the Riemann problem (9) has a unique solution for arbitrary $g(\xi) \in L_{2}\left(\mathbb{T}^{m}\right)$.

\section{DISCRETE BOUNDARY VALUE PROBLEMS IN A HALF-SPACE}

We will consider here certain pseudo-differential operators using general concepts of the theory of special operator equations.

\subsection{Discrete Laplacian}

We define the discrete Laplacian by divided difference of second order instead of partial derivatives

$$
\begin{gathered}
\frac{\partial^{2} u}{\partial x_{k}^{2}} \sim u_{d}\left(x_{1}, \cdots, \tilde{x}_{k}+2, \cdots, x_{m}\right)-2 u_{d}\left(x_{1}, \cdots, \tilde{x}_{k}+1, \cdots, x_{m}\right) \\
+u_{d}\left(x_{1}, \cdots, \tilde{x}_{k}, \cdots, x_{m}\right) \equiv \Delta_{x_{k}}^{2} u_{d},
\end{gathered}
$$

so that

$$
\sum_{k=1}^{m} \frac{\partial^{2} u}{\partial x_{k}^{2}} \equiv \Delta u \sim \sum_{k=1}^{m} \Delta_{x_{k}}^{2} u_{d} \equiv \Delta_{d} u_{d} .
$$

Remark 2. This is not a convolution operator but a difference one in a discrete space.

Applying the discrete Fourier transform to the last expression one can easily obtain the formula

$$
\left(F_{d}\left(\Delta_{d} u_{d}\right)\right)(\xi)=\left(\sum_{k=1}^{m}\left(e^{i \xi_{k}}-1\right)^{2}\right) \tilde{u}_{d}(\xi) .
$$

It is natural the function $\Delta_{d}(\xi) \equiv \sum_{k=1}^{m}\left(e^{i \xi_{k}}-1\right)^{2}$ should be called a symbol of the discrete Laplacian.

Let $A_{d}$ be a linear bounded operator acting in the discrete space $L_{2}\left(\mathbb{Z}^{m}\right)$. A a rule this operator is considered with the following operator equation

$$
A_{d} u_{d}=v_{d}, \quad v_{d} \in L_{2}\left(\mathbb{Z}^{m}\right),
$$

and if the operator is such that one can apply the discrete Fourier transform to the equation (10) reducing it to the multiplier $\sigma_{d}(\xi) \tilde{u}_{d}(\xi)=\tilde{v}_{d}(\xi), \xi \in \mathbb{R}^{m}$, then the function $\sigma_{d}(\xi)$ is called a symbol of operator 
$A_{d}$. Of course $A_{d}$ means not an arbitrary operator but such that above multiplier representation is valid; it may be a convolution operator or a difference one. Let us note in the last equation all functions are periodic, thus we can consider these functions on $\mathbb{T}^{m}$.

Factorization technique for a symbol [3, 9] is used if the equation (10) is considered not on the whole lattice $\mathbb{Z}^{m}$ but on its part, in our case on $\mathbb{Z}_{+}^{m}=\left\{\tilde{x} \in \mathbb{Z}^{m}: \tilde{x}_{m} \geq 0\right\}$. In other words instead of the equation (10) one considers the equation

$$
P_{+} A_{d} u_{d}^{+}=v_{d}^{+}, \quad v_{d}^{+} \in L_{2}\left(\mathbb{Z}_{+}^{m}\right),
$$

where $P_{+}: L_{2}\left(\mathbb{Z}^{m}\right) \rightarrow L_{2}\left(\mathbb{Z}_{+}^{m}\right)$ is a projector, and a solution $u_{d}^{+}$belongs to $L_{2}\left(\mathbb{Z}_{+}^{m}\right)$.

Everywhere below we consider discrete operators with continuous periodic symbols.

Definition 3. The symbol $\sigma_{d}(\xi)$ is called an elliptic symbol if $\inf _{\xi \in \mathbb{T}^{m}}\left|\sigma_{d}(\xi)\right|>0$.

Remark 3. For our case it is equivalent that the symbol is not vanishing everywhere.

\section{PERIODIC FACTORIZATION}

Let us denote $\xi=\left(\xi^{\prime}, \xi_{m}\right)$.

Definition 4. A periodic factorization for elliptic symbol $\sigma_{d}(\xi)$ is called its representation in the form $\sigma_{d}\left(\xi^{\prime}, \xi\right)=\sigma_{d}^{+}\left(\xi^{\prime}, \xi\right) \cdot \sigma_{d}^{-}\left(\xi^{\prime}, \xi\right)$, where $\sigma_{d}^{ \pm}(\xi)$ admit bounded analytic continuation with respect to the variable $\xi_{m}$ into half-strips $\Pi_{ \pm}=\left\{z \in \mathbb{C}: z=\xi_{m}+i \tau, \xi_{m} \in[-\pi, \pi], \pm \tau>0\right\}$ for almost all $\xi^{\prime}$.

Definition 5. An index of periodic factorization of elliptic symbol $\sigma_{d}(\xi)$ is called divided by $2 \pi$ variation of an argument of the function $\sigma_{d}(\xi)$ under varying $\xi_{m}$ from $-\pi$ to $\pi$.

Remark 4. This is easily seen that the index of factorization does not depend on $\xi^{\prime}$.

Since the discrete Laplacian with the symbol $\Delta_{d}(\xi)$ is not an elliptic symbol according the definition 3 we will consider here the discrete Helmholtz operator $H_{d} \equiv \Delta_{d}-k^{2} I$, where $I$ is an identity operator with some fixed $k \in \mathbb{R}$ to satisfy the definition 1 .

Lemma 5. If index of factorization for discrete operator $A_{d}$ is zero then its symbol admits a periodic factorization.

All details for constructing such factorizations are contained in $[12,13]$. Key role plays a periodic analogue of the Hilbert transform, i.e. an operator of the form

$$
\left(H_{\xi^{\prime}}^{p e r} \tilde{u}_{d}\right)(\xi)=\frac{1}{2 \pi i} v \cdot p \cdot \int_{-\pi}^{\pi} \cot \frac{\eta_{m}-\xi_{m}}{2} \tilde{u}_{d}\left(\xi^{\prime}, \eta_{m}\right) d \eta_{m} .
$$

This is a simple corollary of the theory of periodic Riemann boundary value problem which permits in future considering non-vanishing indices.

Lemma 6. Index of factorization for the discrete operator $H_{d}$ is equal to 1.

This fact is verified immediately. Indeed, under fixed $\xi^{\prime}$ the argument varying is determined by the term with $e^{i \xi_{m}}$ for arbitrary $k$.

\section{A GENERAL SOLUTION}

Lemmas 5 and 6 permit describing a structure of a general solution for the equation (11) for the case $A_{d} \equiv H_{d}$ in the space $L_{2}\left(\mathbb{Z}_{+}^{m}\right)$ using methods [3,9] and the theory of periodic Riemann boundary value problem $[12,13]$

Theorem 4. For $A_{d}=H_{d}$ all solutions of the equation (3) are given by the formula

$$
\tilde{u}_{d}(\xi)=e^{-i \xi_{m}} h_{+}^{-1}\left(\xi^{\prime}, \xi_{m}\right)\left(H_{\xi^{\prime}}^{p e r}\left(h_{-}^{-1} \widetilde{l v_{d}^{+}}\right)\right)(\xi)+\tilde{c}\left(\xi^{\prime}\right) e^{-i \xi_{m}} h_{+}^{-1}\left(\xi^{\prime}, \xi_{m}\right),
$$

where $h_{ \pm}\left(\xi^{\prime}, \xi_{m}\right)$ are factors of periodic factorization for the symbol $e^{-i \xi_{m}}\left(\Delta_{d}(\xi)+k^{2}\right), l v_{d}^{+}$is an arbitrary continuation of $v_{d}^{+}$onto the whole $\mathbb{Z}^{m}, c\left(x^{\prime}\right)$ is an arbitrary function from $L_{2}\left(\mathbb{Z}^{m-1}\right)$.

Factors of a periodic factorization are constructed according to classical books of F.D. Gakhov [5] and N.I. Muskhelishvili [6] with the change of the Hilbert transform $H$ by the operator $H_{\xi^{\prime}}^{p e r}$. 


\subsection{Boundary Value Problems}

The Theorem 4 contains an assertion on non-uniqueness of a solution for the equation (11). To extract an unique solution one needs additional conditions. Usually they use boundary conditions. Simplest variants are given by discrete analogues of Dirichlet or Neumann conditions. We will consider here discrete Dirichlet conditions. We will require that a solution of the equation (11) satisfies the following discrete Dirichlet condition

$$
\left.u_{d}\right|_{\tilde{x}_{m}=0}=g_{d}\left(\tilde{x}^{\prime}\right),
$$

where $g_{d}$ is given function of a discrete variable on a discrete hyper-plane $Z^{m-1}$. The condition (12) for Fourier image takes the form

$$
\int_{-\pi}^{\pi} \tilde{u}_{d}\left(\xi^{\prime}, \xi_{m}\right) d \xi_{m}=\tilde{g}_{d}\left(\xi^{\prime}\right)
$$

and according to the Theorem 4 it leads to the following integral equation with respect to $\tilde{c}\left(\xi^{\prime}\right)$

$$
\int_{-\pi}^{\pi} c\left(\xi^{\prime}\right) e^{-i \xi_{m}} h_{+}^{-1}\left(\xi^{\prime}, \xi_{m}\right) d \xi_{m}=\tilde{f}_{d}\left(\xi^{\prime}\right)
$$

we use the notations

$$
\tilde{f}_{d}\left(\xi^{\prime}\right)=\tilde{g}_{d}\left(\xi^{\prime}\right)-\int_{-\pi}^{\pi} e^{-i \xi_{m}} h_{+}^{-1}\left(\xi^{\prime}, \xi_{m}\right) 1 / 2\left(I+H_{\xi^{\prime}}^{p e r}\right)\left(h_{-}^{-1} \widetilde{l v_{d}^{+}}\right)(\xi) d \xi_{m} .
$$

Further, denoting

$$
\int_{-\pi}^{\pi} e^{-i \xi_{m}} h_{+}^{-1}\left(\xi^{\prime}, \xi_{m}\right) d \xi_{m} \equiv b\left(\xi^{\prime}\right)
$$

and supposing $b\left(\xi^{\prime}\right) \neq 0$ one can easily find $\tilde{c}\left(\xi^{\prime}\right)=b^{-1}\left(\xi^{\prime}\right) \tilde{f}_{d}\left(\xi^{\prime}\right)$. Then a solution of the problem (11), (12) for Fourier image takes the form

$$
\tilde{u}_{d}(\xi)=e^{-i \xi_{m}} h_{+}^{-1}\left(\xi^{\prime}, \xi_{m}\right)\left(1 / 2\left(I+H_{\xi^{\prime}}^{p e r}\right)\left(h_{-}^{-1} \widetilde{l v_{d}^{+}}\right)\right)(\xi)+b^{-1}\left(\xi^{\prime}\right) \tilde{f}_{d}\left(\xi^{\prime}\right) e^{-i \xi_{m}} h_{+}^{-1}\left(\xi^{\prime}, \xi_{m}\right),
$$

Remark 5. One can verify that really $b\left(\xi^{\prime}\right)$ is a non-vanishing complex constant.

Theorem 5. Discrete boundary value problem (11), (12) is uniquely solvable in the space $L_{2}\left(\mathbb{Z}_{+}^{m}\right)$ for an arbitrary right-hand side $v_{d} \in L_{2}\left(\mathbb{Z}_{+}^{m}\right)$ and an arbitrary boundary function $g_{d} \in$ $L_{2}\left(\mathbb{Z}^{m-1}\right)$.

\subsection{Discrete Poisson Formula}

If the right-hand side of the equation is a zero, $v_{d}^{+} \equiv 0$, then formula for a solution of the discrete boundary value problem (11), (12) will be very simplified $\tilde{u}_{d}(\xi)=b^{-1}\left(\xi^{\prime}\right) \tilde{g}_{d}\left(\xi^{\prime}\right) e^{-i \xi_{m}} h_{+}^{-1}\left(\xi^{\prime}, \xi_{m}\right)$, and after applying the inverse discrete Fourier transform it will be like this expression

$$
u_{d}\left(\tilde{x}^{\prime}, \tilde{x}_{m}\right)=\sum_{\tilde{y}^{\prime} \in \mathbb{Z}^{m-1}} P_{d}\left(\tilde{x}^{\prime}-\tilde{y}^{\prime}, \tilde{x}_{m}\right) g_{d}\left(\tilde{y}^{\prime}\right)
$$

where the function of a discrete variable $P_{d}(\tilde{x})$ is defined by inverse discrete Fourier transform of the function $b^{-1}\left(\xi^{\prime}\right) e^{-i \xi_{m}} h_{+}^{-1}\left(\xi^{\prime}, \xi_{m}\right)$. The formula (13) is a discrete analogue of the Poisson formula for solving the Dirichlet problem in a half-space. 


\section{CONCLUSION}

Taking into account these results one can wait some interesting interrelations between discrete and continue theory which will permit to justify obtaining discrete solutions with computer calculations for a lot of distinct boundary value problems and wide classes of equations. Moreover such technique are also useful for studying difference equations in multidimensional spaces [10. 11, 14-16]. Last these "discrete" considerations can be transferred on more general situations and operators. It will be a subject of forthcoming papers of the author.

\section{ACKNOWLEDGMENTS}

This work is supported by the State contract of the Russian Ministry of Education and Science (contract no 1.7311.2017/B).

\section{REFERENCES}

1. M. E. Taylor, Pseudodifferential Operators (Princeton Univ. Press, Princeton, 1981).

2. F. Treves, Introduction to Pseudodifferential Operators and Fourier Integral Operators (Springer, New York, 1980).

3. G. Eskin, Boundary Value Problems for Elliptic Pseudodifferential Equations (Am. Math. Soc., Providence, 1981).

4. M. A. Shubin, Pseudodifferential Operators and Spectral Theory (Springer, Berlin, Heidelberg, 2001).

5. F. D. Gakhov, Boundary Value Problems (Dover, New York, 1981).

6. N. I. Muskhelishvili, Singular Integral Equations (North-Holland, Amsterdam, 1976).

7. S. Bochner and W. T. Martin, Several Complex Variables (Princeton Univ. Press, Princeton, 1948).

8. V. S. Vladimirov, Methods of the Theory of Functions of Many Complex Variables (Dover, New York, 2007).

9. V. B. Vasil'ev, Wave Factorization of Elliptic Symbols: Theory and Applications (Kluwer Academic, Dordrecht, 2000).

10. V. B. Vasilyev, "General boundary value problems for pseudo differential equations and related difference equations," Adv. Differ. Equations 289, 1-7 (2013).

11. V. B. Vasilyev, "On some difference equations of first order," Tatra Mt. Math. Publ. 54, 165-181 (2013).

12. A. V. Vasilyev and V. B. Vasilyev, "Discrete singular operators and equations in a half-space," Azerb. J. Math. $3,84-93(2013)$.

13. A. V. Vasilyev and V. B. Vasilyev, "Periodic Riemann problem and discrete convolution equations," Differ. Equations 51, 652-660 (2015).

14. A. V. Vasilyev and V. B. Vasilyev, "On some classes of difference equations of infinite order," Adv. Differ. Equations 211, 1-9 (2015).

15. A. V. Vasilyev and V. B. Vasilyev, "On solvability of some difference-discrete equations," Opusc. Math. 36, 525-539 (2016).

16. A. V. Vasilyev and V. B. Vasilyev, "Difference equations in a multidimensional space," Math. Model. Anal. 21, 336-349 (2016). 